\title{
AKAMAI Project: Teleradiology in the Pacific
}

\author{
Jay F. Cook, Mark F. Hansen, Robert G. Leckie, and James J. Francoise
}

O NE OF THE MOST ambitious and technologically sophisticated automation initiatives being conducted at Tripler Army Medical Center (Honolulu, HI) is called "AKAMAI", a native Hawaiian word meaning intelligent or wise. This is a Congressionally funded, multiyear project, sponsored by Senator Daniel K. Inouye. The project seeks to establish Tripler as the nation's most modern and capable digital telecommunication medical center.

Over the next 5 years, Tripler will make the transition into a fully digital electronic medical environment capable of overcoming the time and distance barriers imposed by the vast expanse of the Pacific Ocean. Using satellite and submarine-cable communications, Tripler will establish contact with relatively underserved customers and patients located in remote clinics and small hospitals on the Pacific Rim. Tripler will be able to project the consultative expertise of its medical specialists and subspecialists into these remote locations and bring medical center level consultation to primary care providers and patients. In many cases, this will obviate the need for costly medical air evacuation of patients eastward across the Pacific to obtain tertiary-level care and treatment.

Sites affected include the US Department of Defense installations scattered around the $\mathrm{Pa}$ cific, whose health care beneficiaries look to Tripler for specialty- and subspecialty-level health care. Ships at sea will be able to access Tripler via telecommunication links. Remote island populations under the US Public Service will also be offered access.

On the Korean Peninsula, access to radiology interpretation services has been a perennial problem of the US Army 18th Medical Command. In the past, as much as a 2-week film-toreport turnaround process was routine. With installation of a teleradiology system, clinicians

From the Department of Radiology, Tripler Army Medical Center, Honolulu, HI, and the US Army Medical Material Agency, Ft Detrick, $M D$.

Address reprint requests to Jay $F$. Cook, MD, Department of Radiology, Tripler Army Medical Center, Honolulu, HI 96859.

Copyright $\odot 1995$ by W.B. Saunders Company

0897-1889/95/0801-1006\$3.00/0 in remote clinics can expect to receive finalized reports within 24 hours. US Naval Hospital (Camp Lester, Okinawa), some 4,000 miles from Tripler, anticipates the loss of its neurosurgeon. Using a combination of teleradiology and telemedicine consultation with Tripler, a neurosurgical replacement is not expected to be required.

On the island of Oahu, Tripler will serve as a hub with an initial five spokes distributed to other Army, Navy, Air Force, and Marine installations on the island. Radiographic images will be interpreted at either the remote site or at Tripler, depending on the availability of a radiologist. A long-term archive will be supported at Tripler and the island military health care system will become predominantly filmless. Data compression, wireless communications and dryprint technologies are being explored to facilitate this transition and enhance the positive aspects of the system.

Operation "Shooting Star," a primary component of the AKAMAI Project, describes Tripler's plan to deploy a sturdy self-contained, selfsupportable medical imaging clinic to distant, remote locations. The imaging clinic will use components of the Deployable Medical System Field Hospital. This system will use filmless radiology technology. Once this deployable telepresence unit (DTU) is airlifted to and positioned in a remote location, Tripler's entire array of tertiary level-care medical specialty and subspecialty expertise can be digitally projected forward, using satellite or ground communications to support ambulatory patient care and emergency casualty-triage operations. Because of the filmless mode of operation, no chemicals or film will be required, simplifying logistics in remote and sometimes harsh environments. Obvious applications of this DTU capability will be for war-fighting contingencies and civilian disaster-relief operations.

Teleradiology alone or in combination with other teleimaging modalities will improve health care in the Pacific Basin by removing time and distance barriers. Further studies will be conducted in areas of compression technology, cost/benefit analysis, and dry-print integration. 